\title{
Loss of imprinting and aberrant methylation of $I G F 2$ in placentas from pregnancies complicated with fetal growth restriction
}

\author{
OURANIA KOUKOURA ${ }^{1}$, STAVROS SIFAKIS ${ }^{1}$, GIANNOULA SOUFLA ${ }^{2}$, APOSTOLOS ZARAVINOS ${ }^{2}$, \\ SOPHIA APOSTOLIDOU ${ }^{3}$, ALLISON JONES ${ }^{3}$, MARTIN WIDSCHWENDTER ${ }^{3}$ and DEMETRIOS A. SPANDIDOS ${ }^{2}$ \\ ${ }^{1}$ Department of Obstetrics and Gynaecology, University Hospital of Heraklion; ${ }^{2}$ Laboratory of Clinical Virology, \\ University of Crete, Heraklion, Crete, Greece; ${ }^{3}$ Institute for Women's Health, University College London, London, UK
}

Received June 17, 2011; Accepted July 13, 2011

DOI: $10.3892 /$ ijmm.2011.754

\begin{abstract}
The objective of this study was to investigate the hypothesis that the altered epigenetic mechanisms that regulate $I G F 2$ imprinting in placentas from fetal growth restricted (FGR) pregnancies affect $I G F 2$ expression leading to impaired fetal growth. We investigated gene transcription, genotyping and the methylation patterns of $I G F 2$ from 31 and 17 placentas from FGR-complicated and normal pregnancies, respectively. A statistically significant decrease in $I G F 2$ mRNA levels was observed in the placentas from the FGR pregnancies. Loss of imprinting (LOI) was only detected in the abnormal placentas. The evaluation of the percentage of the methylated reference (PMR) of two different potentially differentially methylated regions (DMR) demonstrated significant PMR values in both sites for the normal and FGR pregnancies with no significant differences. Our results suggest the involvement of the IGF2 imprinted gene in placental function and fetal growth and the possible association of epigenetic alterations with the pathophysiology of fetal growth restriction.
\end{abstract}

\section{Introduction}

It is well known that fetal growth restriction (FGR) is not a single disorder, but instead has various causes (1). Placental dysfunction ranks high among the most common causes of FGR. Although numerous placental histopathological changes have been described, little is known about the precise etiology and the contribution of placental genes in this disorder $(2,3)$.

Imprinted genes are known to be involved in regulating placental growth and function and are therefore considered to be suitable candidates for a role in FGR development $(4,5)$. Genomic imprinting is the preferential silencing of one copy of an autosomal gene while the other copy is expressed (6). This

Correspondence to: Professor D.A. Spandidos, Laboratory of Virology, Medical School, University of Crete, P.O. Box 1527, Heraklion 710 03, Crete, Greece

E-mail: spandidos@spandidos.gr

Key words: methylation, imprinting, IGF2, placenta, fetal growth restriction parent-of-origin-specific expression is regulated by epigenetic modifications, such as DNA methylation. Imprinted genes that are paternally expressed (maternally imprinted) promote growth of the fetus, while maternally expressed (paternally imprinted) genes act as growth suppressors to ensure the appropriate allocation of limited maternal resources to each offspring (7). Insulin-like growth factor 2 (IGF2) and $H 19$ represent two oppositely expressed genes located adjacent to each other at $11 \mathrm{p} 15.5$ that share the same transcription regulatory epigenetic mechanisms (8). IGF2 is highly expressed in mouse and human placenta and affects both the functional capacity of the placenta to transfer nutrients to the fetus and placental size $(9,10)$. It is expressed in most tissues only from the paternal allele, with the maternal allele being transcriptionally silent.

Imprinting occurs primarily by allelic-specific methylation of cytosines in areas of DNA that are rich in $\mathrm{CpG}$ dinucleotides (11). In most human tissues the imprinting of IGF2 depends on a differentially methylated region (DMR) which is located upstream of $H 19$ promoters (12). This region functions as a methylation-sensitive insulator that binds to a CCCTC factor (CTCF) on the unmethylated maternal allele preventing the interaction of $I G F 2$ with the enhancer located downstream of H19, and partitioning the two alleles into transcriptionally 'silent' and 'active' regions $(11,13)$. Two regions of allele-specific methylation also exist within the human $I G F 2$. The region spanning exons 2 and 3 is homologous to the mouse $\mathrm{dmr} 0$ and another region within exon 9 is similar to mouse $\mathrm{dmr} 2$ (4).

IGF2 expression is characterized by a delicate epigenetic regulation through several promoters exhibiting developmentaldependent expression patterns (14). Aberrant DNA methylation which can modify imprinted gene expression may provide an attractive mechanism linking environmental causes to placental insufficiency and subsequently to the development of intrauterine growth restriction. To investigate the role of the imprinted IGF2 in the pathogenesis of FGR, we aimed to determine whether altered $I G F 2$ expression correlates with relaxed imprinting in FGR placentas. We also examined the epigenetic profiles of the IGF2/H19 domain in these tissues to determine whether deregulated methylation status correlates with $I G F 2$ transcription and potential imprinting deregulation. We postulated that altered epigenetic mechanisms affect $I G F 2$ imprinting and deregulate its expression leading to FGR. 


\section{Materials and methods}

Sample collection. This study was approved by the Research and Ethics Committee of the University Hospital of Heraklion, Crete, Greece. Informed consent was obtained from all patients. Placentas were obtained after vaginal deliveries or caesarean sections from 31 women with singleton pregnancies that where complicated by FGR, as well as from 17 normal pregnancies. The FGR pregnancies were recruited in the immediate intrapartum period. The estimated fetal weight was below the 5th percentile and all pregnancies demonstrated sonographic findings of FGR such as reduced fetal size, abnormal Doppler measurements of the umbilical artery, and/or oligohydramnios (data not shown). Exclusion criteria were multiple pregnancy, chorioamnionitis, chromosomal abnormalities and fetal anatomical defects. Control placentas were obtained from pregnancies with healthy appropriate-for-gestational-age term neonates with birth weight (BW) between the 10th and 90th percentile and no other pregnancy complications. Biopsy specimens were collected from six locations between the decidual and chorionic plates in order to limit tissue heterogeneity (15). Each sample contained the deciduas basalis and villous placenta. Areas involving calcification or infarcts were avoided. Three fragments from each placenta were thoroughly washed in phosphate-buffered $0.9 \%$ saline to minimize blood contamination and subsequently snap-frozen and stored at $-80^{\circ} \mathrm{C}$ for further treatment. Baseline demographic characteristics and medical history information (maternal weight, height, age, parity, smoking, mode of delivery, pregnancy complications, fetal gender, and BW) were recorded. Gestational age at delivery was determined by the mother's last menstrual cycle and/or sonographic measurements at 11-14 weeks of gestation.

DNA and RNA extraction. Genomic DNA and total RNA were extracted from FGR and normal placentas as previously described (16). RNA and DNA concentration was measured on a UV spectrophotometer (Hitachi Instruments Inc., USA).

Reverse transcription and real-time PCR. Reverse transcription reactions for the preparation of first-strand cDNA from $2 \mu \mathrm{g}$ of total RNA were performed using the reverse transcription system as previously described (17), followed by DNase treatment in order to ensure avoidance of genomic DNA contamination. Real-time PCR reactions were carried out on an Mx3000P thermal cycler (Stratagene, La Jolla, CA) using SYBR ${ }^{\circledR}$ Green I Master Mix (Stratagene, Greece) and the primers listed in Table I (18). After initial denaturation at $95^{\circ} \mathrm{C}$ for $10 \mathrm{~min}$, samples were subjected to 40 amplification cycles comprising denaturation at $95^{\circ} \mathrm{C}$ for $30 \mathrm{sec}$, annealing at $65^{\circ} \mathrm{C}$ for $30 \mathrm{sec}$ and elongation at $72^{\circ} \mathrm{C}$ for $30 \mathrm{sec}$, followed by a melt curve analysis in which the temperature was increased from 55 to $95^{\circ} \mathrm{C}$ at a linear rate of $0.2^{\circ} \mathrm{C} / \mathrm{sec}$. Negative controls were included in each PCR reaction. Normalized transcription levels were calculated for each pathological or normal sample using the formula: Normalized sample or Control $=\left(1+\mathrm{E}_{\mathrm{GOI}}\right)^{-\Delta \mathrm{Ct}} /\left(1+\mathrm{E}_{\mathrm{GAPDH}}\right)^{\Delta \mathrm{Ct}}$.

Genotyping of IGF2 polymorphisms. Genotypes of FGR and control tissues were determined by PCR of genomic DNA as previously described (18). Briefly, PCR reactions were performed in a total volume of $25 \mu \mathrm{l}$ containing $5 \mu \mathrm{M}$ of $5 \mathrm{X}$ Green GoTaq ${ }^{\circledR}$
Reaction Buffer, $1.5 \mathrm{mM} \mathrm{MgCl}_{2}, 0.2 \mathrm{mM}$ of each deoxynucleotide triphosphate (dNTPs), $0.6 \mathrm{U}$ of GoTaq Flexi DNA polymerase (Promega, Madison) and 200 ng of genomic DNA or cDNA. PCR conditions were $95^{\circ} \mathrm{C}$ for $90 \mathrm{sec}$, followed by 29 cycles of $95^{\circ} \mathrm{C}$ for $25 \mathrm{sec}$ and optimal annealing temperature for $1 \mathrm{~min}$, and finally $72^{\circ} \mathrm{C}$ for $10 \mathrm{~min}$. The optimal annealing temperature was $65^{\circ} \mathrm{C}$. The PCR products were examined by electrophoresis on a $3 \%$ agarose gel and photographed on a UV transilluminator. Determination of the IGF2 exon 9 bp 819 polymorphism required restriction enzyme digestion with $A p a \mathrm{I}$. PCR products (4-5 $\mu \mathrm{g}$ ) were mixed with 20-30 $\mathrm{U}$ of the appropriate enzyme (Promega, Madison) in a total volume of $20 \mu \mathrm{l}$, and digested at $37^{\circ} \mathrm{C}$ for $\sim 6 \mathrm{~h}$. Products were electrophoresed on $3 \%$ agarose and photographed on a UV transilluminator.

Analysis of DNA methylation. Sodium bisulfite conversion (Zymo Research, Berlin, Germany) and MethyLight analysis (Applied Biosystems, Warrington, UK; Metabion, Munich Germany) were performed as previously described (19). Each MethyLight reaction, at a specific locus, covered on average 5-7 CpG dinucleotides. A detailed list of primer and probes for all analyzed loci is provided in Table I. Briefly two sets of primers and probes, designed specifically for bisulfite-converted DNA, were used: a methylated set for the gene of interest and a reference set (COL2A1) to normalize for input DNA. Specificity of the reactions for methylated DNA was confirmed separately using SssI- (New England Biolabs; www.newenglandbiolabs.co.uk) treated human white blood cell DNA (heavily methylated). The percentage of fully methylated molecules at a specific locus was calculated by dividing the IGF2:COL2A1 ratio of a sample by the IGF2:COL2A1 ratio of the SssI-treated human white blood cell DNA and multiplied by 100 . The abbreviation PMR (percentage of methylated reference) indicates this measurement. The analysis was performed in a blinded manner, and cases and controls were randomly mixed for bisulfite treatment and real-time PCR. The concentration of bisulfite-modified DNA (assessed by the level of the reference gene COL2A1) was the same between the cases and controls.

Statistical analysis. Mean values and standard deviations were calculated for continuous parameters. Data were presented as percentages in case of categorical variables. The distribution of continuous characteristics within two groups of individuals was compared using the non-parametric Wilcoxon rank-sum test. The potential association between categorical variables was examined by the $\chi^{2}$ test. The Spearman's rank correlation coefficient was estimated to assess the relationship between various continuous variables. Moreover, the association between mRNA or PMR values and characteristics of the study participants (BW, gestational age, etc.) was modelled using linear regression analysis. All tests of significance were two-sided, and a $\mathrm{P}<0.05$ indicated significance. Statistical analysis was performed using SPSS 17 statistical package (SPSS, Inc., Chicago, IL).

\section{Results}

Sample characteristics. We analyzed 31 placental samples from FGR-complicated pregnancies and 17 samples from normal pregnancies. BW was below the 5th percentile in all the FGR pregnancies, with almost $90 \%$ of them being below the 3rd 
Table II. Allelic expression of $I G F 2$.

\begin{tabular}{lccc}
\hline Placental samples & Case nos. & gDNA & cDNA \\
\hline $\begin{array}{l}\text { Loss of imprinting } \\
\text { of IGF2 }\end{array}$ & & & \\
FGR & 10 & $\mathrm{~A} / \mathrm{B}$ & $\mathrm{A} / \mathrm{B}$ \\
FGR & 13 & $\mathrm{~A} / \mathrm{B}$ & $\mathrm{A} / \mathrm{B}$ \\
FGR & 16 & $\mathrm{~A} / \mathrm{B}$ & $\mathrm{A} / \mathrm{B}$ \\
FGR & 22 & $\mathrm{~A} / \mathrm{B}$ & $\mathrm{A} / \mathrm{B}$ \\
FGR & 25 & $\mathrm{~A} / \mathrm{B}$ & $\mathrm{A} / \mathrm{B}$ \\
FGR & 37 & $\mathrm{~A} / \mathrm{B}$ & $\mathrm{A} / \mathrm{B}$ \\
FGR & 43 & $\mathrm{~A} / \mathrm{B}$ & $\mathrm{A} / \mathrm{B}$ \\
FGR & 48 & $\mathrm{~A} / \mathrm{B}$ & $\mathrm{A} / \mathrm{B}$ \\
FGR & 98 & $\mathrm{~A} / \mathrm{B}$ & $\mathrm{A} / \mathrm{B}$
\end{tabular}

Maintenance of imprinting of IGF2

\begin{tabular}{lccc} 
FGR & 7 & $\mathrm{~A} / \mathrm{B}$ & $\mathrm{A}$ \\
FGR & 50 & $\mathrm{~A} / \mathrm{B}$ & $\mathrm{B}$ \\
Control & 58 & $\mathrm{~B}$ & \\
FGR & 62 & $\mathrm{~B}$ & \\
Control & 66 & $\mathrm{~A} / \mathrm{B}$ & $\mathrm{A}$ \\
Control & 75 & $\mathrm{~A} / \mathrm{B}$ & $\mathrm{B}$ \\
FGR & 76 & $\mathrm{~B}$ & \\
Control & 79 & $\mathrm{~A} / \mathrm{B}$ & $\mathrm{A}$ \\
Control & 87 & $\mathrm{~A} / \mathrm{B}$ & $\mathrm{B}$ \\
Control & 88 & $\mathrm{~A}$ & \\
FGR & 96 & $\mathrm{~A} / \mathrm{B}$ & $\mathrm{B}$ \\
FGR & 97 & $\mathrm{~A} / \mathrm{B}$ & $\mathrm{A}$ \\
FGR & 99 & $\mathrm{~A} / \mathrm{B}$ & $\mathrm{A}$ \\
Control & 102 & $\mathrm{~A} / \mathrm{B}$ & $\mathrm{A}$ \\
Control & 118 & $\mathrm{~A}$ & \\
Control & 128 & $\mathrm{~A} / \mathrm{B}$ & $\mathrm{A}$ \\
\hline
\end{tabular}

percentile. The percentiles were calculated based on an individually customized fetal and BW percentile method (www. gestation.net). FGR cases and controls were compared with respect to baseline characteristics and outcome. The distributions of maternal age, weight, BMI, fetal gender, parity and smoking were well balanced between the two groups (data not shown). As expected, there were significant differences between gestational age, BW and BW percentiles in the FGR subjects versus controls.

Imprinting analysis of IGF2 in placental tissues. Exon 9 IGF2 polymorphism, which involved the creation of a restriction enzyme site, was evaluated in this study. PCR of genomic DNA with specific primers (Table I), followed by restriction digestion, determined the informative samples. RT-PCR of total RNA allowed for the analysis of the expressed alleles. In total, 16 out of 31 placental FGR samples and 9 out of 17 control placentas were informative (heterozygous) for IGF2 (Table II). In a total of 25 informative cases, LOI was found in 9 specimens, all of which were FGR samples (Fig. 1). Normal placentas did not reveal a biallelic expression of $I G F 2$. Therefore, a statistically 


\section{gDNA}

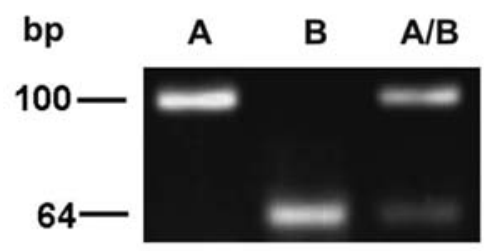

cDNA

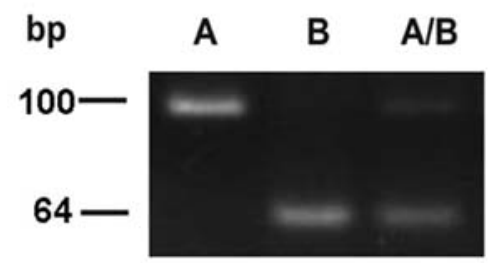

Figure 1. Genotyping and imprinting of the IGF2 gene in the placenta. Representative samples of IGF2 LOI. Upper gel indicates representative samples of ApaI-digested PCR products for genotyping of IGF2. Heterozygous cases were labeled with $\mathrm{A} / \mathrm{B}$, which were considered as informative. Lower gel indicates representative samples of ApaI-digested RT-PCR products for imprinting status analysis. Loss of imprinting was demonstrated in sample labeled A/B. significant difference in the incidence of LOI of IGF2 was demonstrated between the FGR and control placentas $(\mathrm{P}=0.02)$. The remaining 16 informative samples of $I G F 2$ revealed maintenance of imprinting (MOI) in 7 FGR and 9 control samples.

Quantitative analysis of the IGF2 gene. We tested the expression levels of $I G F 2$ in the FGR and control placentas. IGF 2 transcripts derived from FGR placentas were down-regulated compared to controls ( $\mathrm{P}=0.01)$ (Fig. 2A). A linear regression analysis did not reveal a relationship between $I G F 2$ expression and any of the covariates including BW percentile, gestational age at birth, and maternal characteristics.

In order to examine the effect of imprinting defects on expression, we also examined the relative expression of IGF2 in samples that demonstrated aberrant imprinting patterns. Hence, we tested whether there were different mRNA levels in placentas with a biallelic expression of $I G F 2$, compared to those that maintained the imprinting status. Nine FGR samples that demonstrated LOI for IGF2 showed decreased mRNA expression compared to FGR samples that maintained the imprinting pattern; however, this decrease was not significant.

DNA methylation patterns in FGR placentas. We measured the percentage of methylated reference (PMR) of two different potential DMRs (Fig. 3). IGF2 exon 2/3 region from base 65545
A.

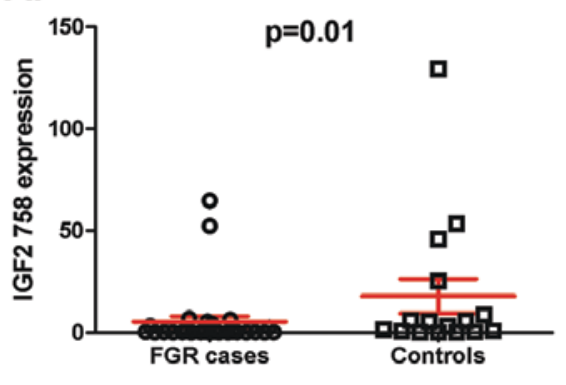

B.

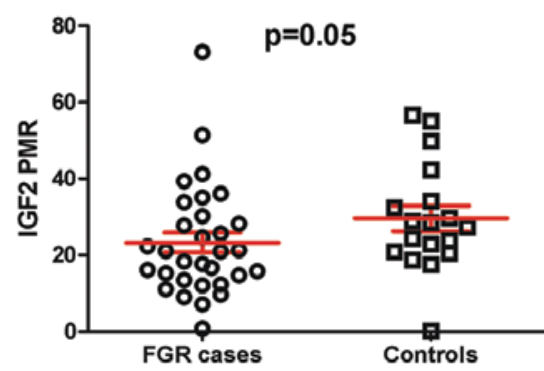

C.

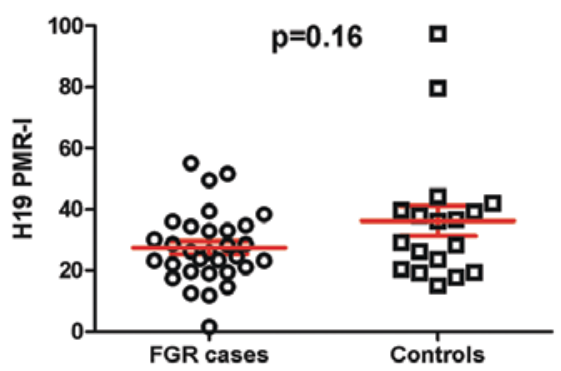

Figure 2. (A) IGF2 transcripts derived from FGR placentas were significantly down-regulated compared to controls ( $\mathrm{P}=0.01)$. Mean values with $\mathrm{SEM}$ are depicted. (B) Percentage of methylated reference (PMR) values in the $I G F 2$ exon $2 / 3$ region. FGR samples demonstrated lower methylation values than controls ( $\mathrm{P}=0.05$ ). Bars indicate mean values and SEM. (C) Percentage of methylated reference (PMR) values in the H19 transcription start site. Bars indicate mean values and SEM. P-values are indicated.

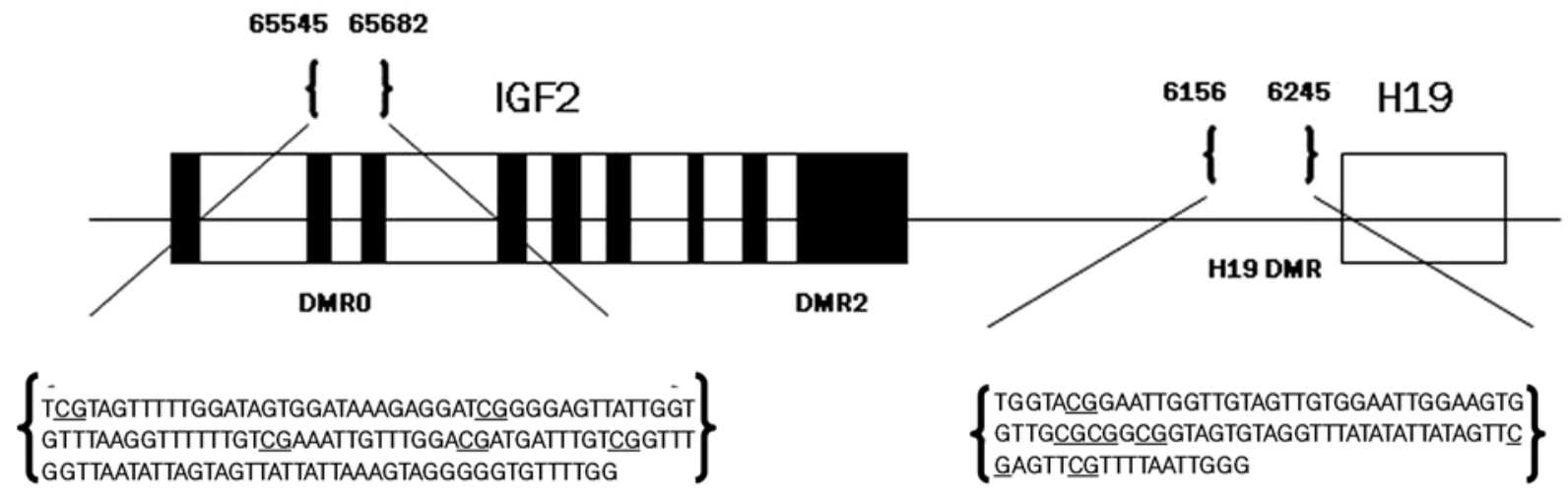

Figure 3. IGF2/H19 imprinted cluster. Black vertical bars indicate $I G F 2$ exons. Location of the PCR primers used are indicated by numbered parentheses. Detailed view of the sequence is presented between brackets. CpG dinucleotide locations are underlined. 

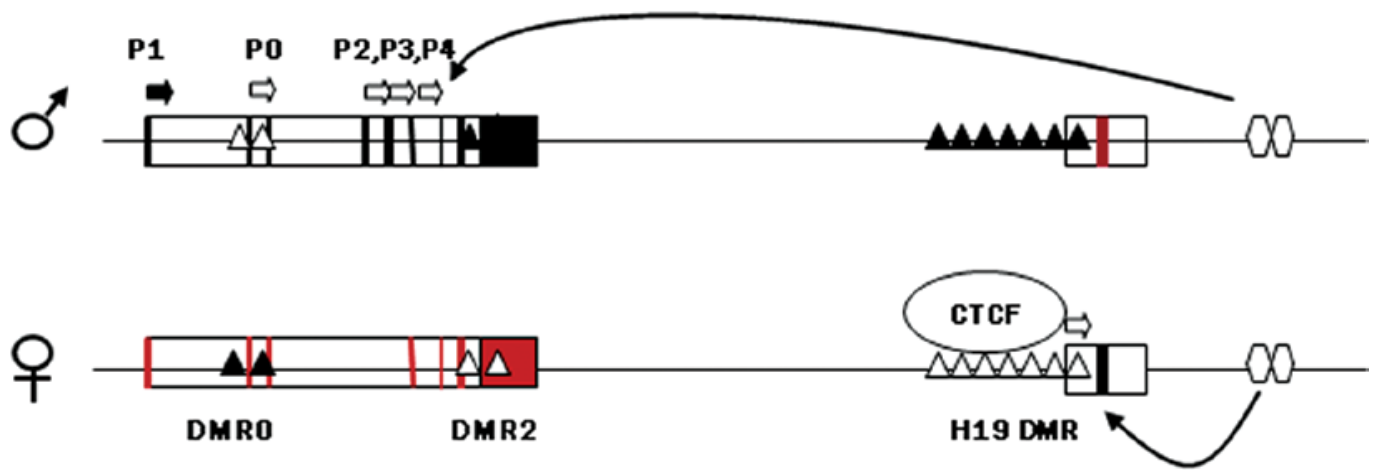

Figure 4. Schematic representation of the imprinted regulation of $I G F 2 / H 19$ cluster. Maternal and paternal chromosomes are indicated. Vertical black bars represent exons from the expressed allele; red vertical bars represent exons from the silenced allele. Black arrows show the direction of transcription (white arrows, active transcription from IGF2 P0, P2, P3, P4 and $H 19$ promoter; black arrow, non-imprinted P1 IGF2 promoter). Ovals represent enhancers. Arrowed lines represent enhancer activity. Three known differentially methylated regions (DMR) are presented as sets of triangles. Solid triangles represent methylated DMRs and clear triangles represent unmethylated regions. DMR upstream $H 19$ harbors the binding sites for CTCF. Methylation of this region on the paternal chromosome prevents binding with CTCF and allows the IGF2 promoter to assess enhancers located downstream of $H 19$. IGF2 is subsequently transcribed. On the maternal chromosome the non-methylated H19 DMR is bound to CTCF insulating the IGF2 promoter from the 3 ' enhancers and allowing the H19 promoter unimpeded access to the enhancers. Maternal H19 is subsequently transcribed. There are also differentially IGF2-methylated regions (DMRO, DMR2). DMR0 is methylated on the maternal allele. Although DMR at exon 9 is methylated on the paternal allele, this region most likely represents a site of tissue-specific methylation which is not involved in $I G F 2$ imprinting.

to 65682 was evaluated in order to determine the methylation patterns of our samples. FGR placentas demonstrated lower methylation references compared to controls, with mean PMR values of 23.3 and $29.6 \%$, respectively, a difference that was marginally significant ( $\mathrm{P}=0.05)$ (Fig. 2B). Methylation levels of the $I G F 2$ promoter did not correlate with gene expression. Additionally, as shown in linear regression analysis, IGF 2 PMR values were not associated with $\mathrm{BW}$ percentiles $(\mathrm{P}=0.17), \mathrm{BW}$ $(\mathrm{P}=0.14)$ and gestational age $(\mathrm{P}=0.2)$. We extended our methylation analysis to the subset of samples that demonstrated LOI for IGF 2 compared to those that maintained monoallelic gene expression. The two groups showed similar methylation values (22.9 vs. $22.8 \%$, respectively).

Methylation status of the region spanning from 6156 to 6245 bp upstream of the H19 transcription start site, which contains CTCF-binding sites (20), was also assessed (Fig. 3). Compared with methylation values of the normal placentas, those from the growth-restricted pregnancies had lower PMR, (mean value 27.4 vs. 36.2\%, P=0.15) (Fig. 2C). H19 PMR measurements of the specific region were not associated with $\mathrm{BW}$ percentiles $(\mathrm{P}=0.52), \mathrm{BW}(\mathrm{P}=0.73)$ or gestational age $(\mathrm{P}=0.8)$ in linear regression analysis. Since methylation of this site controls $I G F 2$ transcription we investigated the impact of methylation on $I G F 2$ mRNA levels. No correlation was noted between the H19 DMR methylation levels and $I G F 2$ expression. Samples with LOI for IGF2 had higher PMR values compared to those with MOI with mean values of 31.3 and $26.1 \%$, respectively. This difference did not reach statistical significance $(\mathrm{P}=0.84)$.

\section{Discussion}

In the present study we investigated the epigenetic characteristics of the IGF2/H19 imprinted domain in FGR placentas in the context of altered IGF2 expression and imprinting. Alterations in the methylation profile of specific regions within the $I G F 2 / H 19$ imprinted domain were detected. We verified the previously reported reduced $I G F 2$ expression noted in growth-restricted placentas. Interestingly a high prevalence of LOI was only noted in the placentas associated with FGR. However, there was a lack of correlation between these two events. Our results highlight the importance of the human chromosome 11p15.5 imprinting cluster in fetal and placental growth and the possible association of epigenetic alterations with the pathophysiology of FGR.

Our results are consistent with previously reported human and animal studies demonstrating a reduced IGF2 mRNA level in placentas from pregnancies with FGR (5,21). IGF2 underexpression results in a reduction in all placental layers and affects the diffusional properties of the placenta (22). Guo et al suggested a correlation between the relative IGF2 expression and BW percentile (21). We were not able to reproduce this observation since there was no association between $I G F 2$ expression and FGR severity in our study.

The biallelic expression of $I G F 2$ was established exclusively in the FGR samples. Similar conclusions were drawn in a study by Yu et al who evaluated the imprinting status of $H 19$ in normal and preeclamptic placentas (23). LOI of H19 was observed only in preeclamptic placentas and was correlated with the severity of the disease (23). Similarly, relaxation of the IGF2 imprinting was demonstrated in tissues with gestational trophoblastic disease unlike the normal placentas (24). Since LOI was also established in the affected placentas in our study, we hypothesize that relaxed IGF2 imprinting might correlate with placental dysfunction which represents a component of the original pathogenetic process of FGR.

Fetal growth restricted samples that displayed LOI had similar IGF2 mRNA levels compared to those with normal imprinting. This observation implies that there is probably no correlation between IGF2 expression and the imprinting profile in the placenta. Diplas et al concluded that even when a correlation between imprinted genes and the pathophysiology of FGR is present, it appears that mechanisms other than imprinting contribute to altered gene transcription (25).

The model of reciprocal IGF2 and $H 19$ expression predicts that the DMR upstream of $H 19$ if methylated in both alleles would result in biallelic expression of IGF2 (20) (Fig. 4). Our 
methylation analysis included the region located upstream of the H19 promoters (Fig. 3). Control and FGR samples demonstrated similar methylation values (36.2 vs $27.4 \%$ ) which corresponds to that previously reported in FGR as well as in control placentas in this region $(21,26)$. There was no correlation between the PMR values of the specific DMR region that we detected and the IGF2 mRNA values.

We compared 9 tissues from the FGR group with LOI and 8 with MOI based on their PMR values. Samples with relaxed imprinting had comparable PMR values compared to those with MOI (31.3 vs. 26.1\%). Increased methylation levels in the placentas with imprinting defects would agree with the above-mentioned model of H19 DMR methylation controlling IGF2 imprinting (Fig. 4). Since we did not demonstrate such a difference, no definite conclusions can be drawn regarding the association of $I G F 2$ imprinting status and methylation patterns in placentas; thus, this event warrants further investigation.

Similarly the difference noted in the IGF2 PMR values between FGR placentas and controls was marginally significant ( 23.3 vs. $29.6 \%, p=0.05$ ). Aberrant methylation of this site has been linked to imprinting defects in cancer as well as in normal tissues (27). Other investigators have postulated that the human DMR0 is a methylation-dependent insulator that regulates $I G F 2$ expression independently of the H19 DMR (28). Since LOI results from failure of the cell to maintain monoallelic expression and can be associated with hypomethylation, we speculated that the reduced trend of IGF2 DMR PMR levels is attributed to the high prevalence of samples with imprinting defects in the FGR group. However, methylation levels of IGF2 DMR in the FGR samples that demonstrated either LOI or MOI in $I G F 2$ were similar. Guo et al found hypomethylation of $I G F 2$ DMR2 in exon 9 in placentas from SGA neonates, but normal methylation values in the H19 DMR for the same group (21). There is no obvious explanation as to why samples with LOI had similar methylation levels in the IGF2 DMR to those with MOI. Since IGF2 DMR methylation patterns are reported to be tissue-specific (4), more data are needed to elucidate these findings.

Several conclusions can be drawn and also several questions can be raised from this study. Whether the observed IGF2 downregulation is a causative factor of FGR or merely a consequence of placental dysfunction remains unexplained. An abnormal biallelic placental expression of imprinted genes has been reported in cases of abnormal placentation such as preeclampsia and gestational trophoblastic disease $(23,24)$. We hypothesize that in FGR cases the placenta responds to chronic hypoperfusion by activating a program of gene expression via genomic imprinting. IGF2 LOI may represent an effort made by the placenta to compensate its reduced functional capacity by leading to a biallelic production of a potent growth factor. The direct effect of IGF2 imprinting in placental tissues is not yet clear.

Our results showed a possible relationship between aberrant DNA methylation and imprinting relaxation that might be associated with FGR. Although we did not include placental weights and histopathological findings, it would be interesting to examine the possible correlation of the above with imprinted gene expression in placental tissues and in umbilical cord blood. Given the complexity of imprinted gene regulation, more studies are needed on the role of imprinted genes in human placenta pathology and for a more effective compre- hension of the relationships between molecular mechanisms and phenotypic events in FGR pregnancies.

\section{Acknowledgements}

This work was supported by a grant awarded by the Hellenic Society of Obstetrics and Gynecology. We wish to thank Dr Dimitrios Koutroulakis for his valuable contribution in tissue sampling and data collection and also George Nikolopoulos for his assistance with the statistical analysis.

\section{References}

1. Lee PA, Chernausek SD, Hokken-Koelega AC and Czernichow P: International Small for Gestational Age Advisory Board. International Small for Gestational Age Advisory Board consensus development conference statement: management of short children born small for gestational age, April 24-October 1,2001. Pediatrics 111: 1253-1261, 2003.

2. Sitras V,Paulssen R, Leirvik J, Vårtun A and Acharya G: Placental gene expression profile in intrauterine growth restriction due to placental insufficiency. Reprod Sci 16: 701-711, 2009.

3. Struwe E, Berzl G, Schild R, et al: Microarray analysis of placental tissue in intrauterine growth restriction. Clin Endocrinol 72: 241-247, 2010.

4. Monk D, Sanches R, Arnaud P, et al: Imprinting of IGF2 P0 transcript and novel alternatively spliced INS-IGF2 isoforms show differences between mouse and human. Hum Mol Genet 15: 1259-1269, 2006

5. McMinn J, Wei M, Schupf N, et al: Unbalanced placental expression of imprinted genes in human intrauterine growth restriction. Placenta 27: 540-549, 2006.

6. Efstratiadis A: Parental imprinting of autosomal mammalian genes. Curr Opin Genet Dev 4: 265-280, 1994.

7. Tycko B and Morison IM: Physiological functions of imprinted genes. J Cell Physiol 192: 245-258, 2002.

8. Steinhoff C, Paulsen M, Kielbasa S, Walter J and Vingron M: Expression profile and transcription factor binding site exploration of imprinted genes in human and mouse. BMC Genomics 10: $144,2009$.

9. Fowden AL, Sibley C, Reik W and Constancia M: Imprinted genes, placental development and fetal growth. Horm Res 65 (Suppl. 3): 50-58, 2006.

10. Randhawa $R$ and Cohen $P$ : The role of the insulin-like growth factor system in prenatal growth. Mol Genet Metab 86: 84-90, 2005.

11. Constancia M, Kelsey GL and Reik W: Resourceful imprinting. Nature 432: 53-57, 2004.

12. Engemann S, Strödicke M, Paulsen M, et al: Sequence and functional comparison in the Beckwith-Wiedemann region: implications for a novel imprinting centre and extended imprinting. Hum Mol Genet 9: 2691-2706, 2000.

13. Kurukuti S, Tiwari VK, Tavoosidana G, et al: CTCF binding at the H19 imprinting control region mediates maternally inherited higher-order chromatin conformation to restrict enhancer access to Igf2. Proc Natl Acad Sci USA 103: 10684-10689, 2006.

14. Brice AL, Cheetham JE, Bolton VN, Hill NC and Schofield PN: Temporal changes in the expression of the insulin-like growth factor II gene associated with tissue maturation in the human fetus. Development 106: 543-554, 1989.

15. Pidoux G, Gerbaud P, Laurendeau I, et al: Large variability of trophoblast gene expression within and between human normal term placentae. Placenta 25: 469-473, 2004.

16. Zaravinos A, Bizakis J and Spandidos DA: Prevalence of human papilloma virus and human herpes virus types 1-7 in human nasal polyposis. J Med Virol 81: 1613-1619, 2009.

17. Koukoura O, Sifakis S, Zaravinos A, et al: Hypomethylation along with increased H19 expression in placentas from pregnancies complicated with fetal growth restriction. Placenta 32: 51-57, 2011.

18. Ulaner GA, Vu TH, Li T, Hu JF, et al: Loss of imprinting of IGF2 and H19 in osteosarcoma is accompanied by reciprocal methylation changes of a CTCF-binding site. Hum Mol Genet 12: 535-549, 2003.

19. Weisenberger DJ, Campan M, Long TI, et al: Analysis of repetitive element DNA methylation by MethyLight. Nucleic Acids Res 33: 6823-6826, 2005. 
20. Hark AT, Schoenherr CJ, Katz DJ, Ingram RS, Levorse JM and Tilghman SM: CTCF mediates methylation-sensitive enhancer blocking activity at the H19/Igf2 locus. Nature 405: 486-489, 2000.

21. Guo L, Choufani S, Ferreira J, et al: Altered gene expression and methylation of the human chromosome 11 imprinted region in small for gestational age (SGA) placentae. Dev Biol 320: 79-91, 2008

22. Sibley CP, Coan PM, Ferguson-Smith AC, et al: Placental-specific insulin-like growth factor 2 (Igf2) regulates the diffusional exchange characteristics of the mouse placenta. Proc Natl Acad Sci USA 101: 8204-8208, 2004.

23. Yu L, Chen M, Zhao D, et al: The $\mathrm{H} 19$ gene imprinting in normal pregnancy and pre-eclampsia. Placenta 30: 443-447, 2009.

24. Kim SJ, Park SE, Lee C, et al: Altered imprinting, promoter usage, and expression of insulin-like growth factor-II gene in gestational trophoblastic diseases. Gynecol Oncol 88: 411-418, 2003 .
25. Diplas AI, Lambertini L, Lee MJ, et al: Differential expression of imprinted genes in normal and IUGR human placentas. Epigenetics 4: 235-240, 2009.

26. Bourque DK, Avila L, Peñaherrera M, von Dadelszen $P$ and Robinson WP: Decreased placental methylation at the H19/ IGF2 imprinting control region is associated with normotensive intrauterine growth restriction but not preeclampsia. Placenta 31: 197-202, 2010.

27. Murphy SK, Huang Z, Wen Y, et al: Frequent IGF2/H19 domain epigenetic alterations and elevated IGF2 expression in epithelial ovarian cancer. Mol Cancer Res 4: 283-292, 2006.

28. Cui H, Onyango P, Brandenburg S, Wu Y, Hsieh CLand Feinberg AP: Loss of imprinting in colorectal cancer linked to hypomethylation of H19 and IGF2. Cancer Res 62: 6442-6446, 2002. 